\title{
ARE COMPTON-THICK AGNs THE MISSING LINK BETWEEN MERGERS AND BLACK HOLE GROWTH?
}

\author{
Dale D. Koceviski ${ }^{1}$, Murray Brightman ${ }^{2}$, Kirpal Nandra ${ }^{3}$, Anton M. Koekemoer ${ }^{4}$, Mara Salvato ${ }^{3}$, James Aird $^{5}$, \\ Eric F. Bell ${ }^{6}$, Li-Ting Hsu ${ }^{3}$, Jeyhan S. Kartaltepe ${ }^{7}$, David C. $\mathrm{Koo}^{8}$, Jennifer M. Lotz ${ }^{4}$, Daniel H. McIntosh ${ }^{9}$, \\ Mark Mozena ${ }^{8}$, David Rosario ${ }^{3}$, and Jonathan R. Trump ${ }^{10}$ \\ ${ }^{1}$ Department of Physics and Astronomy, Colby College, Waterville, ME 04961, USA; dale.kocevski@colby.edu \\ ${ }^{2}$ California Institute of Technology, USA \\ ${ }^{3}$ Max-Planck-Institut für Extraterrestrische Physik, Germany \\ ${ }_{5}^{4}$ Space Telescope Science Institute, USA \\ 5 Institute of Astronomy, Cambridge, UK \\ ${ }^{6}$ University of Michigan, USA
${ }^{7}$ National Optical Astronomy Observatories, USA \\ ${ }^{8}$ University of California, Santa Cruz, USA \\ ${ }^{9}$ University of Missouri, Kansas City, USA \\ ${ }^{10}$ The Pennsylvania State University, USA \\ Received 2015 June 21; accepted 2015 September 9; published 2015 November 23
}

\begin{abstract}
We examine the host morphologies of heavily obscured active galactic nuclei (AGNs) at $z \sim 1$ to test whether obscured super-massive black hole growth at this epoch is preferentially linked to galaxy mergers. Our sample consists of 154 obscured AGNs with $N_{\mathrm{H}}>10^{23.5} \mathrm{~cm}^{-2}$ and $z<1.5$. Using visual classifications, we compare the morphologies of these AGNs to control samples of moderately obscured $\left(10^{22} \mathrm{~cm}^{-2}<N_{\mathrm{H}}<10^{23.5} \mathrm{~cm}^{-2}\right)$ and unobscured $\left(N_{\mathrm{H}}<10^{22} \mathrm{~cm}^{-2}\right)$ AGN. These control AGNs have similar redshifts and intrinsic X-ray luminosities to our heavily obscured AGN. We find that heavily obscured AGNs are twice as likely to be hosted by late-type galaxies relative to unobscured AGNs $\left(65.3_{-4.6}^{+4.1} \%\right.$ versus $\left.34.5_{-2.7}^{+2.9} \%\right)$ and three times as likely to exhibit merger or interaction signatures $\left(21.5_{-3.3}^{+4.2} \%\right.$ versus $\left.7.8_{-1.3}^{+1.9} \%\right)$. The increased merger fraction is significant at the $3.8 \sigma$ level. If we exclude all point sources and consider only extended hosts, we find that the correlation between the merger fraction and obscuration is still evident, although at a reduced statistical significance $(2.5 \sigma)$. The fact that we observe a different disk/spheroid fraction versus obscuration indicates that the viewing angle cannot be the only thing differentiating our three AGN samples, as a simple unification model would suggest. The increased fraction of disturbed morphologies with obscuration supports an evolutionary scenario, in which Compton-thick AGNs are a distinct phase of obscured super-massive black hole (SMBH) growth following a merger/interaction event. Our findings also suggest that some of the merger-triggered SMBH growth predicted by recent AGN fueling models may be hidden among the heavily obscured, Compton-thick population.
\end{abstract}

Key words: galaxies: active - galaxies: evolution - X-rays: galaxies

\section{INTRODUCTION}

Observations over the past two decades have revealed a tight correlation between the mass of a galaxy's stellar bulge and its central super-massive black hole (SMBH; Magorrian et al. 1998; Gebhardt et al. 2000; Tremaine et al. 2002; Gültekin et al. 2009; McConnell \& Ma 2013). This finding is commonly interpreted as evidence that the growth of SMBHs and their host spheroids is connected. Given the effectiveness of violent galaxy mergers to dissipate angular momentum, mergers have long been proposed as a means to forge this connection (Sanders et al. 1988; Hernquist 1989; Kauffmann \& Haehnelt 2000). In this scenario, the strong gravitational torques produced as a result of a merger funnel gas to the center of a galaxy, triggering both accretion onto the central black hole and star formation that grows the stellar bulge (Barnes \& Hernquist 1991; Mihos \& Hernquist 1996). Coupled with selfregulated black hole growth (i.e., active galactic nuclei, AGN, feedback; Di Matteo et al. 2005; Hopkins et al. 2005, 2006), galaxy mergers provide an attractive mechanism to trigger AGN activity and help explain the co-evolution observed between SMBHs and their host galaxies.

However, observational attempts to tie AGN activity to galaxy mergers have produced mixed results. Gas-rich mergers are observed to fuel a substantial fraction of bright quasars (e.g., Guyon et al. 2006; Bennert et al. 2008; Veilleux et al. 2009; Koss et al. 2010, 2012; Satyapal et al. 2014) and recent studies of kinematic galaxy pairs have demonstrated that nuclear activity is indeed enhanced in galaxies with an interacting companion (Ellison et al. 2011; Silverman et al. 2011). On the other hand, morphological studies have consistently found that the bulk of the AGN population does not appear to be triggered by major galaxy mergers. Both at $z \sim 1$ (Grogin et al. 2005; Pierce et al. 2007; Cisternas et al. 2011; Villforth et al. 2014) and more recently at $z \sim 2$ (Schawinski et al. 2011; Kocevski et al. 2012; Rosario et al. 2015), studies have found that X-ray selected AGN hosts are no more likely to exhibit morphological disturbances compared with similar inactive galaxies. In fact, results from the CANDELS survey (Grogin et al. 2011; Koekemoer et al. 2011) indicate that roughly half of moderate-luminosity $\left(L_{\mathrm{X}}<10^{43} \mathrm{erg} \mathrm{s}^{-1}\right)$ AGN at $z \sim 2$ reside in disks and are likely fueled stochastically by secular processes and/or disk instabilities rather than major mergers (Kocevski et al. 2012).

While the efficiency of stochastic fueling is expected to increase with redshift, given the rapid rise in the gas fraction of galaxies at $z>1$ (see e.g., Tacconi et al. 2010), AGN fueling models predict that only a small fraction $(\sim 30 \%)$ of the overall AGN luminosity density and BH mass density are the result of 
this fueling mode (Hopkins et al. 2014). Instead, the majority of $\mathrm{SMBH}$ growth is predicted to be the result of merger-induced fueling, especially at high luminosities $\left(L_{\mathrm{X}}>10^{44} \mathrm{erg} \mathrm{s}^{-1}\right.$; Hopkins \& Hernquist 2009; Draper \& Balantyne 2012). The low merger fraction observed among X-ray selected AGNs out to $z \sim 2$ appears to be at odds with this prediction.

A major caveat associated with these findings is that heavily obscured AGNs are not well sampled by X-ray surveys (see, e.g., Treister et al. 2004). The most obscured, Compton-thick AGNs (hereafter, CT-AGNs) are hidden by extreme column densities $\left(N_{\mathrm{H}}>10^{24} \mathrm{~cm}^{-2}\right)$ of obscuring gas that can absorb even hard X-ray photons. Analysis of the diffuse X-ray background indicates that a significant fraction (up to $\sim 50 \%$ ) of AGN are hidden behind Compton-thick obscuration (Comastri et al. 1995; Ueda et al. 2003; Gilli et al. 2007; Akylas et al. 2012) ${ }^{11}$; however, much remains unknown about the demographics of their host galaxies. In the evolutionary sequence of Sanders et al. (1988), heavily obscured AGNs represent a key phase in the life cycle of galaxies, as it is during this period that SMBHs are predicted to accrete the bulk of their mass (e.g., Fabian 1999; Gilli et al. 2007; Treister et al. 2009; Draper \& Ballantyne 2010). Furthermore, hydrodynamical merger simulations predict that this obscured phase should coincide with the most morphologically disturbed phase of a galaxy interaction (Cattaneo et al. 2005; Hopkins et al. 2008). It is, therefore, acutely possible that past studies have systematically missed the AGN-merger connection by not sampling the obscured AGN population well.

Several studies have attempted to overcome this bias by selecting AGNs at mid-infrared (IR) wavelengths, where radiation absorbed by obscuring circumnuclear dust is expected to be re-emitted (e.g., Lacy et al. 2004; Stern et al. 2005; Daddi et al. 2007; Donley et al. 2007; Soifer et al. 2008). However, the most recent works that have examined the morphologies of IR-selected AGN have produced conflicting results. Schawinski et al. (2012) examined the morphologies of $24 \mu \mathrm{m}$ -selected Dust Obscured Galaxies (DOGs) at $z \sim 2$, a high fraction of which are thought to host heavily obscured AGN based on X-ray stacking analyses (Fiore et al. 2008; Treister et al. 2009). The authors reported a high disk fraction (90\%) and a relatively low merger fraction $(4 \%)$ that is consistent with studies of more unobscured AGN hosts (i.e., Schawinski et al. 2011). On the other hand, J. L. Donley et al. (2015, in preparation) found that galaxies with a power-law spectral slope in the mid-IR, a signature of hot dust near an obscured AGN's central engine (Donley et al. 2007, 2012), have a higher fraction of disturbed morphologies compared with X-ray detected AGN hosts that do not exhibit similar IR emission. This might be the result of the power-law technique preferentially selecting relatively high luminosity AGN, which may be more associated with galaxy mergers (e.g., Draper \& Ballantyne 2012; Treister et al. 2012).

In this study, we re-examine the connection between AGN obscuration and host morphology using a sample of heavily obscured AGNs identified by their X-ray spectral properties. Due to the differential absorption of hard and soft X-ray photons, the shape of an AGN's X-ray spectrum reveals not only the presence of gas along the line of sight, but it also provides a measure of its column density. CT-AGNs, in particular, can be identified by their X-ray spectra due to

\footnotetext{
11 Studies of resolved X-ray sources estimate a Compton-thick fraction of $35 \%-40 \%$ at $z>1$ (Brightman \& Ueda 2012; Buchner et al. 2015).
}

nuclear emission that is Compton scattered into our line of sight even when the direct emission is suppressed. This "reflected" emission has a characteristic spectral shape consisting of a flat continuum and a high equivalent width $\mathrm{Fe} \mathrm{K} \alpha$ fluorescence line (Reynolds et al. 1994; Matt et al. 1996).

Identifying CT-AGNs using low energy $(<10 \mathrm{keV})$ observations from Chandra or XMM-Newton is challenging because the heavy attenuation suffered at these wavelengths often restricts the accuracy of any X-ray spectral analysis. In addition, CT-AGNs often appear softer than expected at low energies due to their reflection-dominated emission. As a result, a simple absorbed power-law fit to the soft X-ray spectra of CT-AGNs will systematically underestimate their obscuring column density, as was recently shown using NuSTAR observations (Gandhi et al. 2014; Lansbury et al. 2015). However, with sufficiently deep observations and proper spectral modeling, even the most obscured CT-AGNs can be identified with relatively soft X-ray observations (e.g., Brightman et al. 2014; Buchner et al. 2014). Several studies have successfully employed X-ray spectral modeling to identify heavily obscured AGNs using both deep Chandra (Tozzi et al. 2006; Georgantopoulos et al. 2009; Alexander et al. 2011; Feruglio et al. 2011; Gilli et al. 2011; Brightman et al. 2014; Buchner et al. 2014) and XMM-Newton observations (Comastri et al. 2011; Georgantopoulos et al. 2013; Lanzuisi et al. 2015).

For this work, we examine the host morphologies of the CTAGN sample of Brightman et al. (2014). These sources were identified using the new spectral models of Brightman \& Nandra (2011) that correctly account for emission from Compton scattering, the geometry of the absorbing material, and include a self-consistent treatment for $\mathrm{Fe} \mathrm{K} \alpha$ emission. These models include all of the signatures of Compton-thick obscuration in a single model, allowing for the identification of CT-AGNs in lower signal-to-noise data than previously possible. Using visual classifications, we examine whether heavily obscured AGN exhibit an enhancement of merger and/ or interaction signatures relative to their unobscured counterparts with the same intrinsic X-ray luminosity and redshift.

Our analysis is presented as follows. In Section 2 we describe the X-ray and optical data used for the study, and discuss the methodology employed to select our sample of obscured AGNs and unobscured control AGNs. The details of our morphological classification scheme are given in Section 3, and our primary results are presented in Section 4. We discuss the implications of our findings in Section 5. Finally, our conclusions are summarized in Section 6. When necessary, the following cosmological parameters are used: $H_{0}=$ $70 \mathrm{~km} \mathrm{~s}^{-1} \mathrm{Mpc}^{-1} ; \Omega_{\mathrm{tot}}, \Omega_{\Lambda}, \Omega_{m}=1,0.3,0.7$.

\section{OBSERVATIONS AND SAMPLE SELECTION}

\subsection{X-Ray Data sets}

The AGN sample used for our analysis is drawn from Chandra data sets in three fields: the Chandra Deep Field South (CDFS; Alexander et al. 2003; Xue et al. 2011), the AEGIS-XD data set in the Extended Groth Strip (EGS; Nandra et al. 2015), and the C-COSMOS observations (Elvis et al. 2009; Civano et al. 2011). These data sets have characteristic exposure times of $4 \mathrm{~ms}, 800 \mathrm{ks}$, and $180 \mathrm{ks}$, and cover an area of roughly $0.13,0.28,0.98$ degrees $^{2}$, respectively. This combination of deep and wide survey data was chosen to ensure that both moderate and high luminosity 


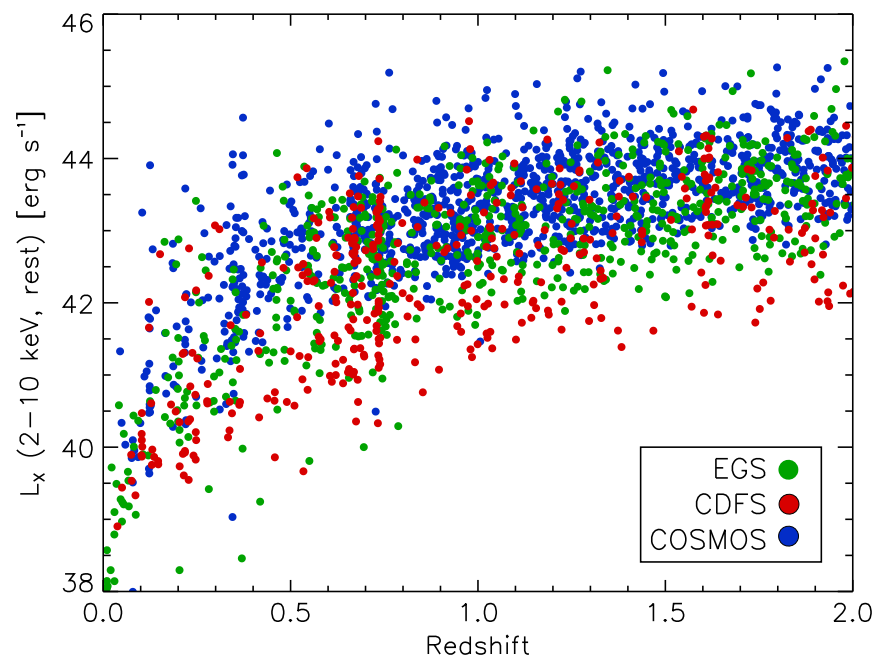

Figure 1. Redshift vs. luminosity for X-ray sources detected in our three target fields. X-ray luminosities are intrinsic rest frame 2-10 keV luminosities, having been corrected for absorption (i.e., setting $N_{\mathrm{H}}=0$ in our best-fit spectral model). The use of both deep and wide survey data allows us to probe a wide range of luminosities, including sources at $L_{X}>10^{44} \mathrm{erg} \mathrm{s}^{-1}$, which are not well sampled in deep/narrow surveys such as the CDFS 4Ms observations.

$\left(L_{\mathrm{X}} \sim 10^{43-45} \mathrm{erg} \mathrm{s}^{-1}\right)$ AGNs are well represented in our final sample.

X-ray source catalogs in the CDFS and AEGIS-XD were created by processing the Chandra observations in each field with the custom reduction and source detection pipeline of Laird et al. (2009). These catalogs were matched to optical counterparts using the maximum-likelihood technique described by Sutherland \& Saunders (1992). In the CDFS, the X-ray sources were cross-matched to the CANDELS $F 160 W$-selected photometry catalog of Guo et al. (2013), while the AEGIS-XD sources were matched to the $3.6 \mu \mathrm{m}$ selected multi-waveband photometric catalog provided by the Rainbow Cosmological Surveys Database (Barro et al. 2011a, 2011b). For the C-COSMOS data set, we adopt the published X-ray source and counterpart catalog of Civano et al. (2012). These published counterparts were identified by cross-matching the X-ray source catalog to the I-band optical sample of Capak et al. (2007) and the $3.6 \mu \mathrm{m}$ sample of Sanders et al. (2007).

Redshifts for the identified X-ray counterparts were drawn from various spectroscopic data sets in each field. For the CDFS, we used the compilation of Cardamone et al. (2010) and Xue et al. (2011). For the EGS field, spectroscopic redshifts are drawn primarily from the DEEP2 (Newman et al. 2013) and DEEP3 (Cooper et al. 2012) redshift surveys. For sources without spectroscopic redshifts in these fields, we use photometric redshifts from $\mathrm{Hsu}$ et al. (2014) and Nandra et al. (2015), which are derived through spectral energy distribution (SED) fitting that employs a combination of galaxy and AGN templates to account for non-stellar emission (e.g., Salvato et al. 2011). For the C-COSMOS sources, we adopt the spectroscopic and photometric redshifts compiled by Civano et al. (2012); the former are drawn primarily from Lilly et al. (2009), Trump et al. (2009), and Brusa et al. (2010), while the latter come from the work of Salvato et al. (2011). The redshift and luminosity distribution of the resulting AGN sample in all three fields is shown in Figure 1.

\subsection{Optical High-resolution Imaging}

To analyze the host morphologies of our AGN sample, we make use of the high resolution Hubble Space Telescope (HST) Advanced Camera for Survey (ACS) optical imaging that is publicly available in each of our three fields. In the CDFS, we use the F850LP ( $z$-band) imaging from the Great Observatories Origins Deep Survey (GOODS; Giavalisco et al. 2004), which covers the central $10^{\prime} \times 16^{\prime}$ of the field. This imaging has an exposure time of $\sim 18200 \mathrm{~s}$ and reaches a limiting magnitude of $m_{A B}=28.3(5 \sigma$, point source, within a circular aperture of radius 0.' 12; Grogin et al. 2011). In COSMOS, we use the $F 814 W\left(I_{F 814 W}\right.$-band $)$ mosaic, which covers an area of roughly $77^{\prime} \times 77^{\prime}$ with an exposure time of $\sim 2000 \mathrm{~s}$ and reaches a limiting magnitude of $m_{A B}=27.2$ (Koekemoer et al. 2007). In the EGS, we make use of the AEGIS $F 814 W$ mosaic, which covers a 10 . $1 \times 70$. 5 region and has an exposure time of $\sim 2100 \mathrm{~s}$ and reaches a limiting magnitude of $m_{A B}=27.5$ (Davis et al. 2007). The ACS imaging in all three fields has a pixel scale of 0. ". $03 /$ pixel.

\subsection{Identifying Obscured AGNs}

We select obscured AGNs from our parent sample using an $\mathrm{X}$-ray spectral analysis that provides a measure of the line of sight obscuration present in each source. The details of this spectral fitting are presented in Brightman et al. (2014); below we briefly summarize this analysis.

Individual source spectra were extracted using ACIS Extract (Broos et al. 2010) and lightly grouped with a minimum of one count per bin using the HEASARC tool grppha. The spectral fitting was carried out with XSPEC using the Cash statistic (c-stat; Cash 1979). The spectral models we use are from Brightman \& Nandra (2011) and employ Monte Carlo simulations to account for Compton scattering and the geometry of the obscuring material. They also include a selfconsistent treatment of iron $\mathrm{K} \alpha$ emission and describe spherical and torus distributions of the circumnuclear material.

Four model combinations are fit to each spectrum. The first three represent obscured emission with various torus geometries. In these models, the column density, $N_{\mathrm{H}}$, primary powerlaw index, $\Gamma$, and power-law normalization are free parameters. Rather than attempting to constrain the torus opening angle from the spectra, three different cases where tested where torus opening angles were fixed at $60^{\circ}, 30^{\circ}$, and $0^{\circ}$; here $0^{\circ}$ is essentially a $4 \pi$ spherical distribution. For opening angles $>0^{\circ}$, we include a secondary power-law component, $\Gamma_{\text {scatt }}$, in the fit, which represents the intrinsic scattered emission reflected by hot electrons filling the cone of the torus. Here $\Gamma_{\text {scatt }}$ is set to the primary power-law index. When the opening angle is $0^{\circ}$, we do not include this scattered component because this model represents a case where there is no escape route for the primary radiation to be scattered into the line of sight. The fourth model is a simple power-law model with two free parameters: the power-law index, $\Gamma$, and its normalization, which represents purely unobscured X-ray emission.

Each of the four model combinations is fit to the source spectrum in turn with at least 100 iterations. We adopted a critical $\Delta$ c-stat of $1 \times 10^{-5}$ as the minimum decrease in the fit statistic required for $\mathrm{XSPEC}$ to say that it has found the minimum. The best-fitting model combination is chosen to be that which presents the lowest c-stat value after penalizing the more complex models (i.e., those with more free parameters). 

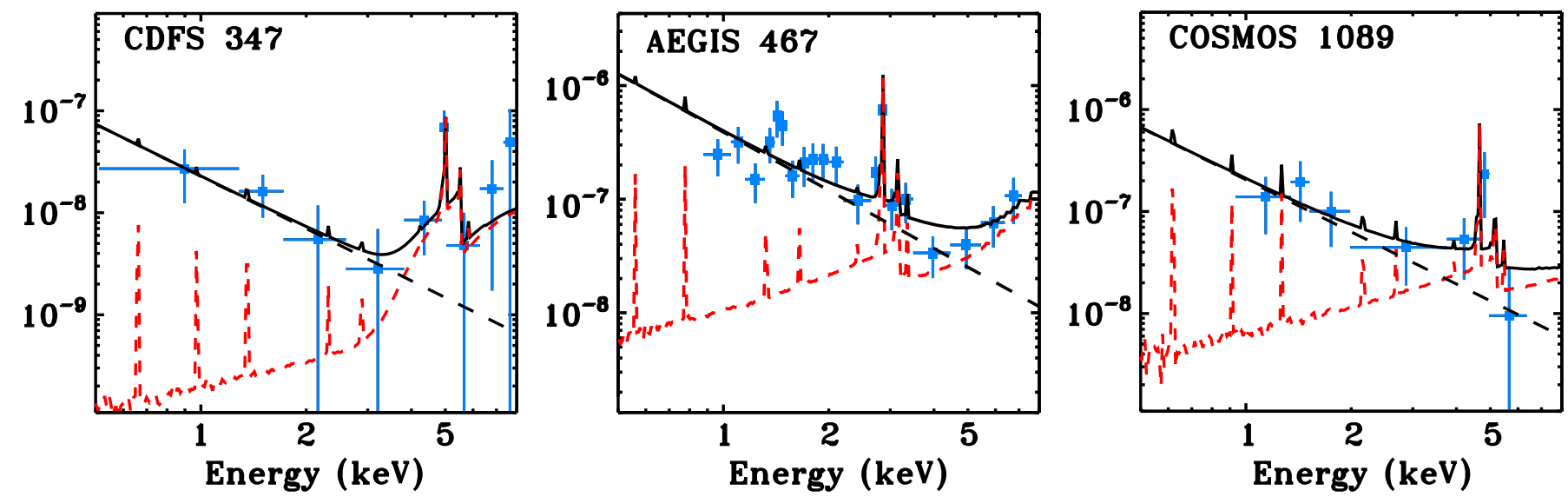

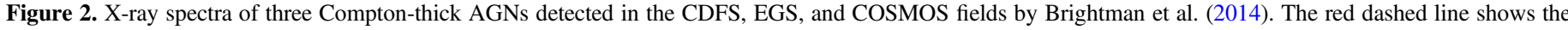

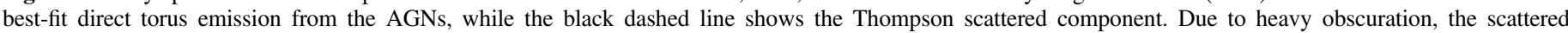

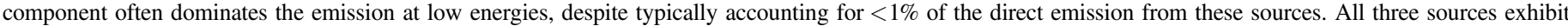
strong Fe $\mathrm{K} \alpha$ emission characteristic of a Compton-thick AGN.

However, Brightman \& Ueda (2012) have shown that for sources with less than 600 counts, large uncertainties in the spectral fits can be reduced by fixing the power-law index in the fit. Thus, for these sources, we use a fixed value of $\Gamma=1.7$, as the mean spectral index of sources with more than 600 counts. We do, however, allow a consideration for intrinsically steep or flat spectra: If the best-fitting model for sources with less than 600 counts, where $\Gamma$ is free, is a significantly better fit than the best-fitting model where $\Gamma$ is fixed using the criterion of $\Delta$ c-stat $>2.71$, we choose the model with $\Gamma$ free. In total, the fraction of sources where $\Gamma$ is left free is 221/549, 220/ 937, and 232/1761 in the CDFS, EGS, and COSMOS fields, respectively. Examples of our X-ray spectral fits in all three fields can be seen in Figure 2.

From our spectral fits we obtain a best-fit line of sight column density, $N_{\mathrm{H}}$, for each source in our parent sample. The resulting distribution of $N_{\mathrm{H}}$ versus redshift and luminosity is shown in Figure 3. As reported in Brightman et al. (2014), we find that heavily obscured $\left(N_{\mathrm{H}}>10^{24} \mathrm{~cm}^{-2}\right)$ sources are best fit by the torus models with opening angles of $30^{\circ}$ or $60^{\circ}$ (i.e., not the $0^{\circ}$ model), whereas sources with $10^{23} \mathrm{~cm}^{-2}$ $<N_{\mathrm{H}}<10^{24} \mathrm{~cm}^{-2}$ are better fit by the $0^{\circ}$ model. This is mostly due to the models being degenerate below $10^{24} \mathrm{~cm}^{-2}$, so the best-fit model was the simplest one (i.e., the $0^{\circ}$ model). Furthermore, for Compton-thin sources, we find that the $N_{\mathrm{H}}$ values obtained using our torus models are in very good agreement with the result obtained by Lanzuisi et al. (2013), who used simple absorption models, as would be expected for Compton-thin sources.

For our morphology study, we define a primary sample of heavily obscured AGNs as those sources with $z<1.5$ and $N_{\mathrm{H}}>10^{23.5} \mathrm{~cm}^{-2}$. We have chosen a column density limit that is lower than the canonical cutoff for Compton-thick AGNs $\left(N_{\mathrm{H}}>1.5 \times 10^{24} \mathrm{~cm}^{-2}\right)$ in order to increase our sample size of heavily obscured AGNs. In addition, our upper redshift limit is motivated by the fact that only $21 \%$ of the heavily obscured AGNs at $z>1.5$ in our three target fields have been imaged with $H S T$ /WFC3. Without this near-infrared imaging, we can not properly access the rest frame optical morphology of galaxies beyond $z \sim 1.5$. Therefore, we limit our analysis to those sources at $z<1.5$ that fall within the EGS, GOODS, and COSMOS HST/ACS imaging.

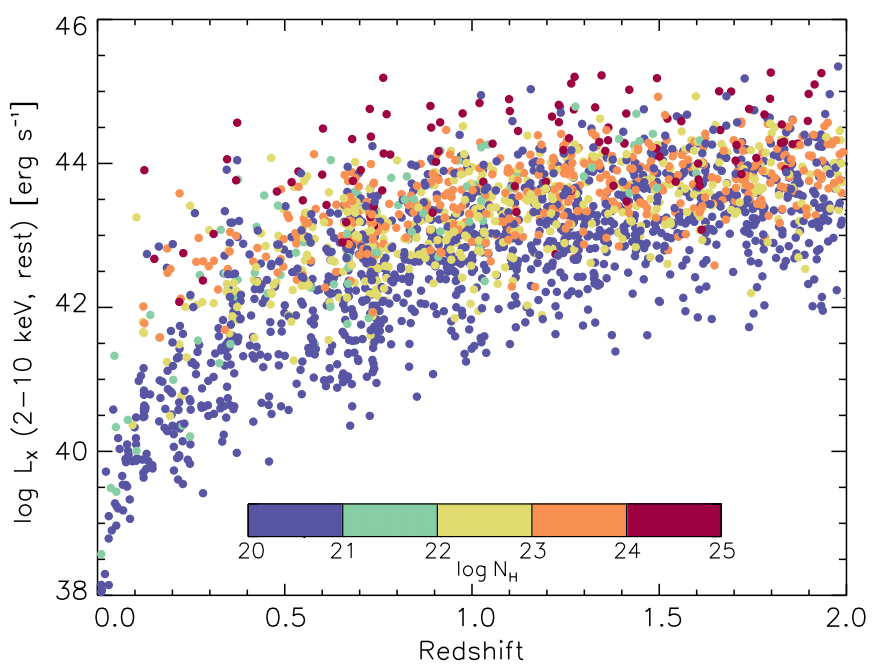

Figure 3. Redshift vs. luminosity for X-ray sources in the CDFS, EGS, and COSMOS fields. Sources are color coded by their level of nuclear obscuration, as determined by our X-ray spectral modeling. X-ray luminosities are intrinsic rest frame $2-10 \mathrm{keV}$ luminosities having been corrected for absorption.

Using these selection criteria results in a sample of 154 heavily obscured AGNs at $z<1.5$ in our three target fields. For simplicity, we refer to these sources as our CT-AGN sample for the remainder of the paper, despite our relaxed $N_{\mathrm{H}}$ cut. Of this sample, $21 \mathrm{CT}$-AGNs are drawn from CDFS data, while 44 and 89 are detected in the EGS and COSMOS fields, respectively.

\subsection{Control Sample Selection}

In order to compare the host morphologies of CT-AGN to that of their less obscured counterparts, we constructed two control samples that are matched in redshift and X-ray luminosity to the CT-AGN sample, but have lower measured absorbing column densities. These two control samples consist of unobscured AGNs with $N_{\mathrm{H}}<10^{22} \mathrm{~cm}^{-2}$ and moderately obscured AGNs with $10^{22}<N_{\mathrm{H}}<10^{23.5} \mathrm{~cm}^{-2}$. In order to match the redshift and luminosity distributions of the samples, for each CT-AGN we randomly select two unobscured and two moderately obscured AGNs that have a redshift within $\Delta z=0.1$ and an absorption-corrected X-ray luminosity within 


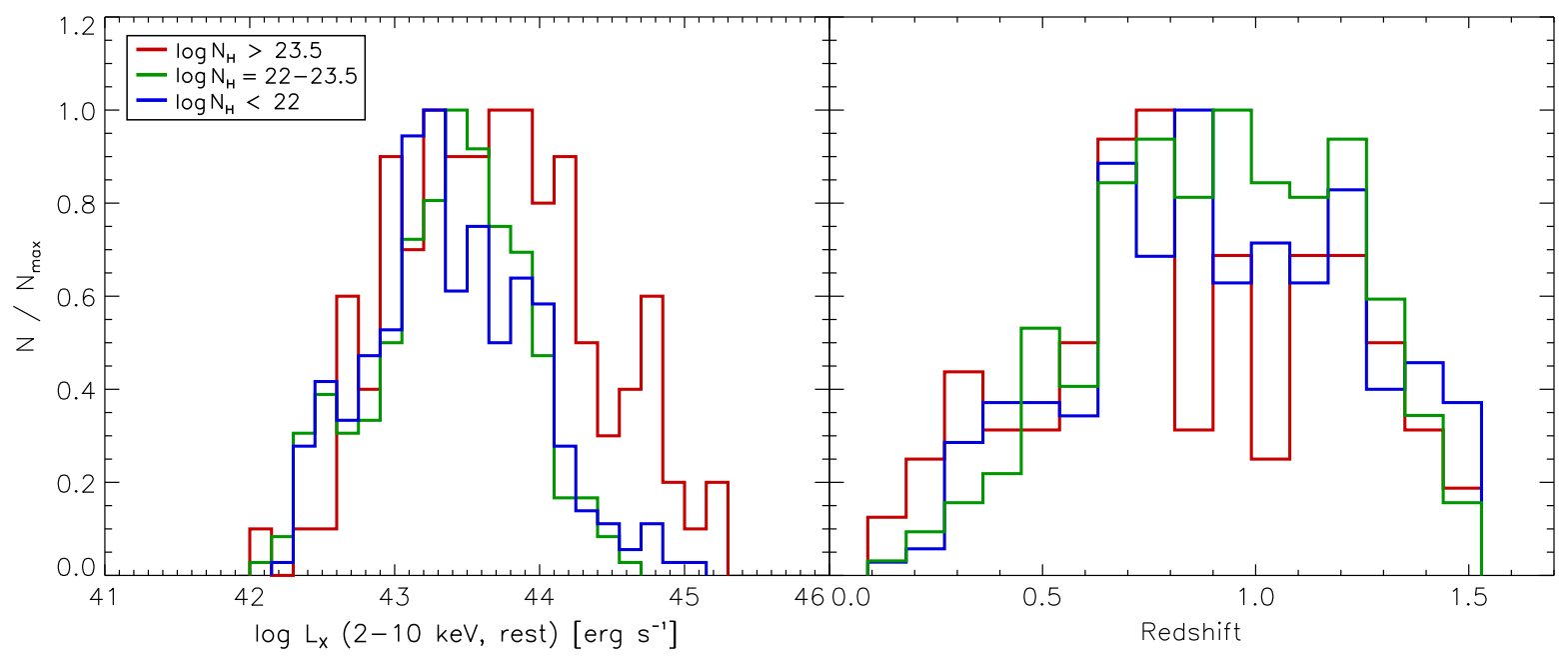

Figure 4. Distribution of absorption corrected, rest frame $2-10 \mathrm{keV}$ luminosities (left) and redshifts $($ right $)$ of the CT-AGN sample $\left(N_{\mathrm{H}}>10^{23.5} \mathrm{~cm}^{-2}\right)$ and our control samples of moderately obscured $\left(10^{22} \mathrm{~cm}^{-2}<N_{\mathrm{H}}<10^{23.5} \mathrm{~cm}^{-2}\right)$ and unobscured $\left(N_{\mathrm{H}}<10^{22} \mathrm{~cm}^{-2}\right)$ AGNs.

a factor of two $\left(0.5 \leqslant L_{\mathrm{X}, \mathrm{CT}} / L_{\mathrm{X}, \text { control }} \leqslant 2\right)$ of the CT-AGNs. For this matching we use rest frame $2-10 \mathrm{keV}$ absorptioncorrected luminosities, which are derived by setting $N_{\mathrm{H}}=0$ in our best-fit spectral model. If two unique comparison AGN could not be found within this parameter range, the search range is iteratively increased by $10 \%$. Because of differences in the depth of the GOODS-S, EGS, and COSMOS HST/ACS imaging, the control AGNs were selected separately for each region. The resulting luminosity and redshift distribution of the three subsamples are shown in Figure 4. The median obscuration-corrected luminosity of the CT-AGN, moderately obscured, and unobscured subsamples are $\left\langle L_{2-10 \mathrm{kev}}\right\rangle=10^{43.69}$, $10^{43.40}$, and $10^{43.34} \mathrm{erg} \mathrm{s}^{-1}$, respectively.

Selecting control AGNs matched in luminosity is challenging because the large absorption corrections applied to the luminosities of the CT-AGNs make them among the most luminous sources in our fields. Statistically (i.e., according to a $\mathrm{K}-\mathrm{S}$ test), the luminosity distributions of the three subsamples are not perfectly matched, with the CT-AGNs having a longer tail toward higher X-ray luminosities, as evidenced by their slightly higher median 2-10 keV luminosity. However, our methodology effectively ensures that we have selected the most luminous moderately obscured and unobscured sources in each field that have similar redshifts as the CT-AGNs. The only way to improve our luminosity matching would be to increase the sample size of AGN available to draw upon. A proper redshift and luminosity matching is vital because it has been proposed that galaxy mergers play a greater role in triggering luminous AGNs, while secular processes trigger lower luminosity AGNs (e.g., Triester et al. 2012). That said, we do not believe the difference in the median luminosity of the three subsamples is large enough to be the primary driver of the results presented in Section 4, because we find no systematic trend between disturbed morphologies and absorption-corrected luminosity among the CT-AGNs that would indicate that mergers dominate the tail of the CT-AGNs luminosity distribution.

Finally, because we are interested in assessing the morphology of the AGN hosts, we follow Cisternas et al. (2011) and apply a magnitude cut of $I_{F 814 W}<24$ for AGN in the EGS and COSMOS fields and $z_{F 850 L P}<24$ for sources in the CDFS. This leaves 120, 279, and 282 sources in the
Compton-thick, moderately obscured, and unobscured AGN samples, respectively. ${ }^{12}$

\section{MORPHOLOGY CLASSIFICATION}

Host morphologies of the CT-AGNs and control AGNs were assessed through visual inspection using a classification scheme similar to the one presented in Kocevski et al. (2012) and Kartaltepe et al. (2015). These inspections were carried out by the lead author, D.K., and performed blind using the reddest HST/ ACS bands available in each field, namely the $F 814 \mathrm{~W}$ band in the EGS and COSMOS fields and the $F 850 L P$ band in the CDFS. In addition, $F 606 \mathrm{~W}$ imaging was used to provide supplemental color information for sources in the EGS and CDFS (similar imaging is not available in the COSMOS field). The size of each thumbnail image was set to cover roughly $100 \mathrm{kpc}$ on a side at the redshift of each AGN, and ranged from 12"-16". Because of differences in the bands used in each field and the depth of the available imaging, control AGNs were drawn from the same field as their matched CT-AGN and the subsequent classifications were carried out separately for each field. ${ }^{13}$

For each AGN host, we classified the morphological type of the galaxy and the degree to which it is disturbed. The possible morphologies were Disk, Spheroid, Irregular/Peculiar, Pointlike. These classes are mutually exclusive, and only the predominate morphology of each galaxy was noted. For example, disk galaxies with a substantial bulge component would simply be classified as disks in this system. This differs from the scheme used in Kocevski et al. (2012), where bulge and disk dominated late-type galaxies were differentiated. The change was made to mitigate the effects of moderate AGN contamination, which can mimic an increase in the bulge-todisk ratio of a galaxy. In this scheme, as long as an extended disk is visible, regardless of the level of nuclear AGN

\footnotetext{
12 Our $N_{\mathrm{H}}$ cut of $10^{23.5} \mathrm{~cm}^{-2}$ was chosen to ensure that roughly half $(61 / 120)$ of our final CT-AGN sample has $N_{\mathrm{H}}>10^{24} \mathrm{~cm}^{-2}$, and are therefore truly Compton-thick. It should be noted, however, that given the large uncertainties on our $N_{\mathrm{H}}$ estimates, even sources with $N_{\mathrm{H}} \sim 10^{23.5} \mathrm{~cm}^{-2}$ could still be consistent with being Compton-thick.

13 Where possible, we have compared the classification of D.K. to those of the CANDELS collaboration (where each galaxy was inspected by an average of four unique classifiers; Kartaltepe et al. 2015), and we find excellent (>90\%) agreement between the two.
} 


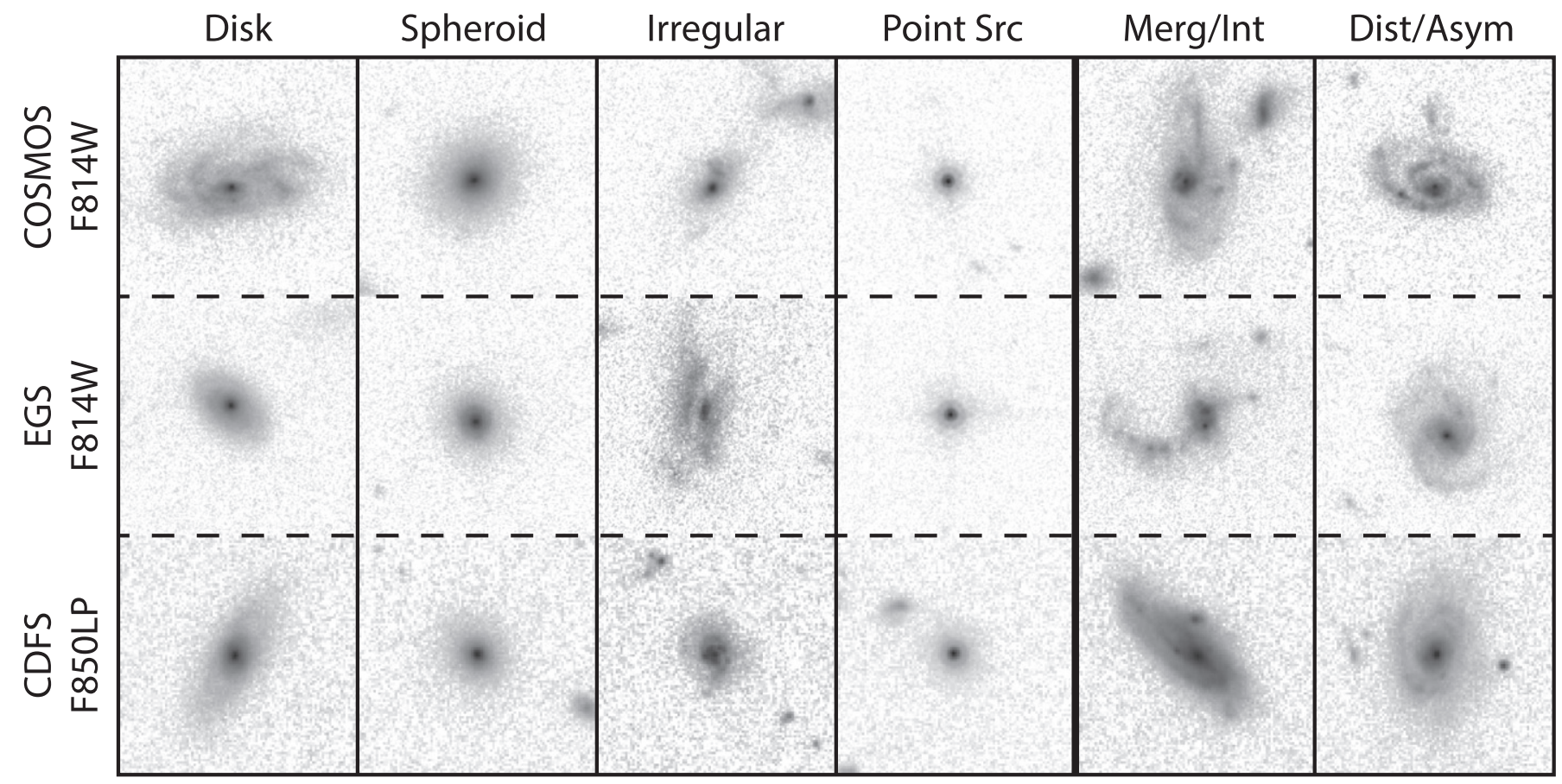

Figure 5. Examples of AGN host galaxies in each morphology and disturbance class of our visual classification scheme. While the Disk, Spheroid, Irregular, and Point-like classifications are mutually exclusive, the Disturbed/Asymmetric class is a superset of the Merger/Interaction class because it includes train-wreck mergers and galaxies that exhibit only minor disturbances. See Section 3 for details.

contamination, the galaxy is classified as a Disk. This is physically motivated by the fact that disks are easily destroyed in major mergers and take a considerable amount of time to reform (Robertson et al. 2006). Therefore the presence of a disk constrains, to a certain extent, the past merger history of a given galaxy. ${ }^{14}$

To gauge the degree to which a galaxy is disturbed, three disturbance classifications were used:

1. Merger/Interaction: two distinct galaxies showing interaction features such as tidal arms or a single train-wreck system exhibiting strong distortions.

2. Disturbed/Asymmetric: all galaxies in the Merger/Interaction class plus single asymmetric or disturbed galaxies with no visible interacting companion.

\section{Undisturbed: none of the above.}

In this scheme, the Merger/Interaction class includes trainwreck mergers that have multiple nuclei and/or strong distortions in a single coalescing system, as well as disturbed galaxies with an interacting companion. The Disturbed/ Asymmetric class, however, serves as a more liberal selection of galaxies that may have experienced an interaction in the recent past. This class includes any galaxy that has a distorted or asymmetric light profile, even those with no visible interacting companion. As a result, these classes are not mutually exclusive, and are similar to the Disturbed I and Disturbed II classes used in Kocevski et al. (2012),

\footnotetext{
${ }^{14}$ It is important to note that while disks can reform following a merger under the right circumstances (when they are sufficiently gas rich and have favorable initial orbital parameters), disk survival is most efficient at low galaxy masses and generally requires conditions that suppress strong inflows toward the galaxy center (see Springel \& Hernquist 2005; Robertson et al. 2006; Hopkins \& Hernquist 2009). This effectively prevents strong bulge growth and AGN fueling; the opposite of the regime we are interested in here.
}

respectively. Examples of AGN host galaxies in each of our morphology classes can be seen in Figure 5.

It should be noted that unresolved AGN hosts (those classified as having Point-like morphologies) are by definition classified as Undisturbed in this system. As a result, any AGN subsample that has a high Point-like fraction will also have a high Undisturbed fraction. Alternatively, one could argue that the disturbance level of Point-like sources is not measurable and should not be classified as Undisturbed. In the following section, we present our results using both approaches: first including Point-like/Undisturbed sources in our analysis and then excluding them completely. While our primary results do not change, the statistical significance of our findings does change as a result of having fewer AGNs in our final sample.

\section{RESULTS}

The fraction of AGN hosts in each of our morphology classes versus their level of nuclear obscuration is shown in Figure 6 and listed in Table 1. The error bars on each fraction reflect the $68.3 \%$ binomial confidence limits given the number of sources in each category, which was calculated using the method of Cameron (2010). For our sample of CT-AGNs we find that $65.3_{-4.6}^{+4.1} \%$ have predominately disk-like morphologies. This includes disks with and without a central bulge. A smaller fraction, $16.5_{-2.8}^{+3.9} \%$, are classified as spheroidal, whereas $12.4_{-2.4}^{+3.6} \%$ are found to have peculiar or irregular morphologies such that neither a prominent disk nor spheroidal component could be discerned. Only a small fraction, $5.0_{-1.3}^{+2.8} \%$, of the CTAGNs are classified as point-like. This may be expected if heavy nuclear obscuration is blocking emission from the central engine in these sources.

For our control sample of unobscured AGNs $\left(N_{\mathrm{H}}<10^{22} \mathrm{~cm}^{-2}\right)$, we find a lower disk fraction $\left(34.5_{-2.7}^{+2.9} \%\right)$ relative to the CT-AGN hosts, a slightly higher spheroid 


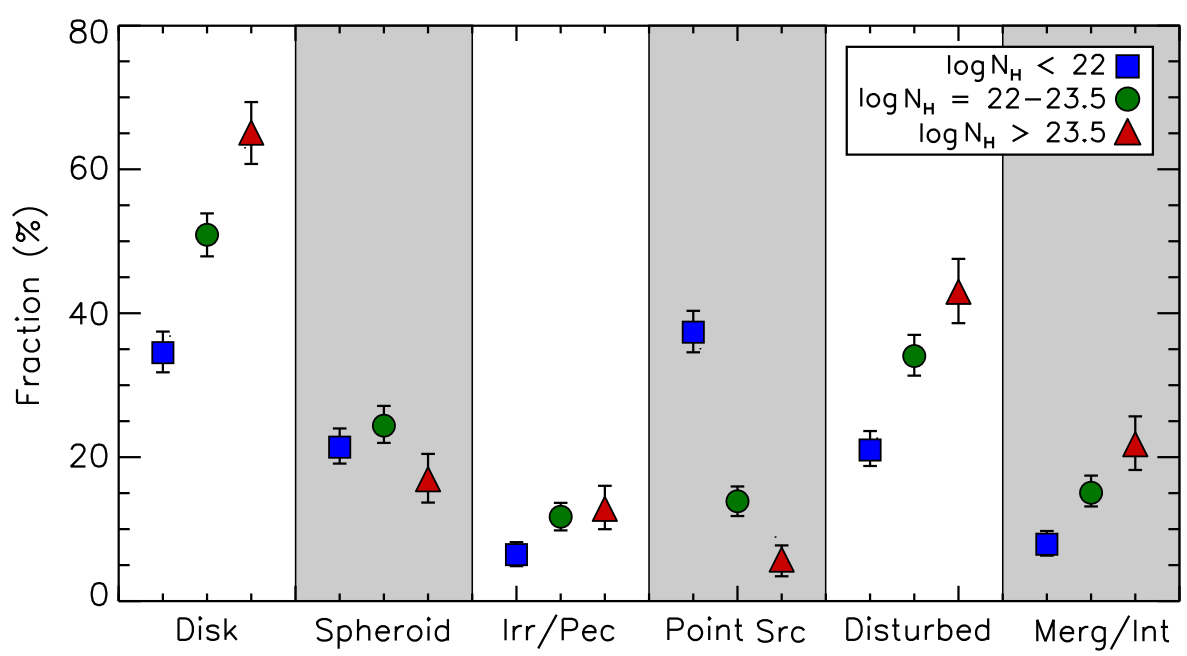

Figure 6. Fraction of AGN hosts at $0.5<z<1.5$ assigned to various morphology and disturbance classes as a function of their nuclear obscuration. We find that the hosts of heavily obscured AGNs are more likely to be disks and have disturbed morphologies relative to the hosts of unobscured AGNs with the same redshift and absorption-corrected X-ray luminosity.

Table 1

Visual Classification Results

\begin{tabular}{|c|c|c|c|c|c|c|}
\hline \multirow{2}{*}{$\frac{\text { Classification }}{\text { Disk }}$} & \multicolumn{2}{|c|}{$\begin{array}{c}\text { Unobscured AGN } \\
\left(N_{\mathrm{H}}<10^{22} \mathrm{~cm}^{-2}\right) \\
\text { All Hosts } / \text { Extended Hosts }\end{array}$} & \multicolumn{2}{|c|}{$\begin{array}{c}\text { Moderately Obscured AGN } \\
\left(10^{22}<N_{\mathrm{H}}<10^{23.5} \mathrm{~cm}^{-2}\right) \\
\text { All Hosts / Extended Hosts }\end{array}$} & \multicolumn{2}{|c|}{$\begin{array}{l}\text { Compton-thick AGN } \\
\left(N_{\mathrm{H}}>10^{23.5} \mathrm{~cm}^{-2}\right) \\
\text { All Hosts/Extended Hosts }\end{array}$} \\
\hline & $34.5_{-2.7}^{+2.9} \%$ & $55.1_{-3.8}^{+3.6} \%$ & $50.9_{-3.0}^{+3.0} \%$ & $58.9_{-32}^{+3.1} \%$ & $65.3_{-4.6}^{+4.1} \%$ & $68.7_{-4.6}^{+4.0} \%$ \\
\hline Spheroid & $21.4_{-2.2}^{+2.6} \%$ & $34.1_{-3.4}^{+3.7} \%$ & $24.4_{-2.4}^{+2.7} \%$ & $28.2_{-2.7}^{+3.0} \%$ & $16.5_{-2.8}^{+3.9} \%$ & $17.4_{-3.0}^{+4.1} \%$ \\
\hline Irregular & $06.4_{-1.2}^{+1.8} \%$ & $10.2_{-1.9}^{+2.7} \%$ & $11.5_{-1.6}^{+2.2} \%$ & $13.3_{-1.9}^{+2.5} \%$ & $12.4_{-2.4}^{+3.6 \%}$ & $13.0_{-2.5}^{+3.8} \%$ \\
\hline Point-like & $37.4_{-2.8}^{+3.0} \%$ & $\ldots$ & $13.6_{-1.8}^{+2.3} \%$ & $\cdots$ & $05.0_{-1.3}^{+2.8} \%$ & $\cdots$ \\
\hline Disturbed/Asym & $21.0_{-2.2}^{+2.6} \%$ & $33.5_{-3.4}^{+3.7} \%$ & $34.1_{-2.7}^{+2.9} \%$ & $39.4_{-3.1}^{+3.2} \%$ & $43.0_{-4.4}^{+4.6} \%$ & $45.2_{-4.5}^{+4.7} \%$ \\
\hline Merger/Interaction & $07.8_{-1.3}^{+1.9} \%$ & $12.5_{-2.1}^{+2.9} \%$ & $15.1_{-1.9}^{+2.4} \%$ & $17.4_{-2.2}^{+2.7} \%$ & $21.5_{-3.3}^{+4.2} \%$ & $22.6_{-3.4}^{+4.3} \%$ \\
\hline
\end{tabular}

fraction $\left(21.4_{-2.2}^{+2.6} \%\right)$, and a lower irregular fraction $\left(6.4_{-1.2}^{+1.8} \%\right)$. Unlike their heavily obscured counterparts, a much larger fraction of the unobscured sources appear point-like in the ACS imaging, accounting for $37.4_{-2.8}^{+3.0} \%$ of the host morphologies. The hosts of the moderately obscured $\left(10^{22} \mathrm{~cm}^{-2}<N_{\mathrm{H}}<\right.$ $10^{23.5} \mathrm{~cm}^{-2}$ ) control sample have morphologies that lie between the two extremes of the Compton-thick and unobscured AGNs. Here, disks, spheroids, and irregulars make up $50.9_{-3.0}^{+3.0} \%$, $24.4_{-2.4}^{+2.7} \%$, and $11.5_{-1.6}^{+2.2} \%$. of the population, respectively. We find an increased point-like fraction $\left(13.6_{-1.8}^{+2.3} \%\right)$ relative to the CT-AGN population; however, this fraction is lower than that found in the unobscured control sample.

The fraction of AGNs with disturbed morphologies in each of our three subsamples is also shown on the right side of Figure 6. We find a statistically significant increase in the Merger/Interaction fraction versus AGN obscuration, rising from $7.8_{-1.3}^{+1.9} \%$ among the unobscured AGNs to $15.1_{-1.9}^{+2.4} \%$ for the moderately obscured AGNs and $21.5_{-3.3}^{+4.2} \%$ for the CT-AGN sample. The increase in the merger fraction of the CT-AGN relative to their unobscured counterparts is significant at the $3.8 \sigma$ level. If we include any galaxy that has a distorted or asymmetric light profile, the overall disturbed fraction increases in all three samples, but the trend with obscuration remains. The Disturbed/Asymmetric fraction increases from $21.0_{-2.2}^{+2.6} \%$ among the unobscured AGNs to $34.1_{-2.7}^{+2.9} \%$ for the moderately obscured AGNs and $43.0_{-4.4}^{+4.6} \%$ for the CT-AGN sample. Here the difference in the disturbed fraction of the
CT-AGN relative to the unobscured AGNs is significant at the $4.4 \sigma$ level.

As discussed in Section 3, the high point source fraction among the unobscured AGNs may artificially drive the disturbed fraction down for that subsample because unresolved hosts are classified as Undisturbed by default. To account for this, we excluded all unresolved hosts from our analysis and present the resulting morphology and disturbance fractions in Figure 7 . When considering only extended hosts, we find that the disk fraction of the three subsamples is in much greater agreement, although the CT-AGNs are still found in disk hosts more often than the unobscured AGNs. We find the disk fraction steadily increases from $55.1_{-3.8}^{+3.6} \%$ among the unobscured AGN to $58.9_{-3.2}^{+3.1} \%$ for the moderately obscured AGN and $68.7_{-4.6}^{+4.0} \%$ for the CT-AGN sample. This trend reverses for the spheroid fraction, which steadily decreases with obscuration. Here the spheroid fraction decreases from $34.1_{-3.4}^{+3.7} \%$ among the unobscured AGN to $28.2_{-2.7}^{+3.0} \%$ for the moderately obscured AGN and $17.4_{-3.0}^{+4.1} \%$ for the CT-AGN sample. The irregular fraction is low for all three subsamples and consistent with showing no trend with obscuration.

The fraction of AGN in extended hosts that exhibit a morphological disturbance is shown in Figures 8 and 9. The correlation between merger fraction and obscuration is still evident when excluding point sources; however, the statistical significance of the increase drops from $3.8 \sigma$ to $2.5 \sigma$. The Merger/Interaction fraction is now $12.5_{-2.1}^{+2.9} \%, 17.4_{-2.2}^{+2.7} \%$, and 


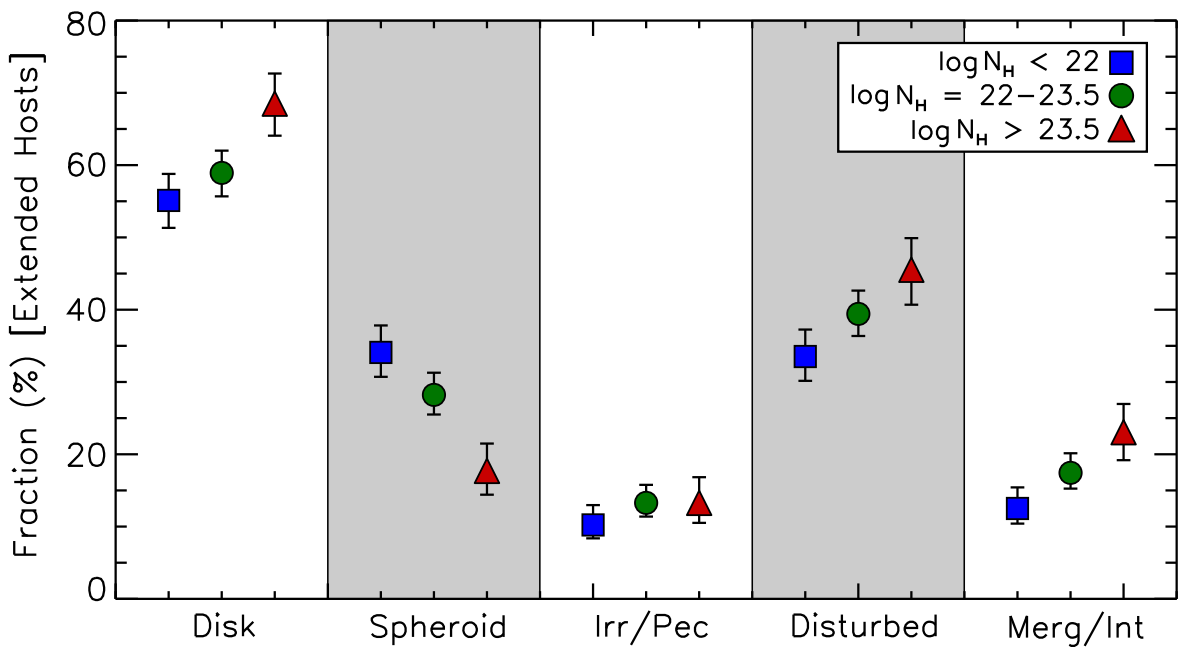

Figure 7. Fraction of extended AGN hosts at $0.5<z<1.5$ assigned to various morphology and disturbance classes as a function of their nuclear obscuration. This plot is the same as Figure 7; however, point sources are now excluded from the analysis. We again find that the hosts of heavily obscured AGN are more likely to be disks and show some morphological disturbance relative to the unobscured control sample, albeit at a reduced statistical significance. See Section 4 for details.



Figure 8. Fraction of AGN hosts in the Disturbed/Asymmetric class as a function of nuclear obscuration with point sources included (open box) and excluded (filled box) from the analysis. In both cases, the hosts of heavily obscured AGN are more likely to be classified as disturbed relative to unobscured AGN with similar redshifts and luminosities. However, when point sources are excluded the statistical significance of this difference drops from $4.4 \sigma$ to $2.3 \sigma$.

$22.6_{-3.4}^{+4.3} \%$, for the unobscured, moderately obscured, and CTAGN samples, respectively. A similar trend is found for the Disturbed/Asymmetric fraction, which increases from $33.5_{-3.4}^{+3.7} \%$ among the unobscured AGN to $39.4_{-3.1}^{+3.2} \%$ for the moderately obscured AGN and $45.2_{-4.5}^{+4.7} \%$ for the CT-AGN sample. Here the statistical significance of the increase is now $2.3 \sigma$.

In summary, we find an increasing disk fraction and decreasing spheroid fraction with increasing nuclear obscuration among AGN at $0.5<z<1.5$. In addition, we find that the fraction of AGN with disturbed host morphologies increases as a function of obscuration. This increase is found whether we consider only train-wreck mergers and galaxies with clear interacting companions or any galaxy showing an asymmetric light profile. It is also present regardless of whether we exclude unresolved host galaxies from our analysis, albeit at a reduced statistical significance.



Figure 9. Fraction of AGN hosts in the Merger/Interaction class as a function of nuclear obscuration with point sources included (open box) and excluded (filled box) from the analysis. In both cases, the hosts of heavily obscured AGN are more likely to be classified as being involved in a merger or interaction relative to unobscured AGN with similar redshifts and luminosities. However, when point sources are excluded the statistical significance of this difference drops from $3.8 \sigma$ to $2.5 \sigma$.

\section{DISCUSSION}

Using a sample of heavily obscured AGN identified by their $\mathrm{X}$-ray spectral signatures, we find a correlation between disturbed host morphology and nuclear obscuration at fixed AGN luminosity and redshift. In this section we discuss the implications of this result in terms of both the AGN unification model (Section 5.1) and the role that mergers play in fueling SMBH growth (Section 5.2). In addition, we conclude the section with a discussion of several important caveats to keep in mind when interpreting our findings (Section 5.3).

\subsection{Implications for the AGN Unification Model}

The standard unification paradigm invokes a torus-like structure that obscures the central engine for some sight lines and not for others, producing the two observed AGN types. In 

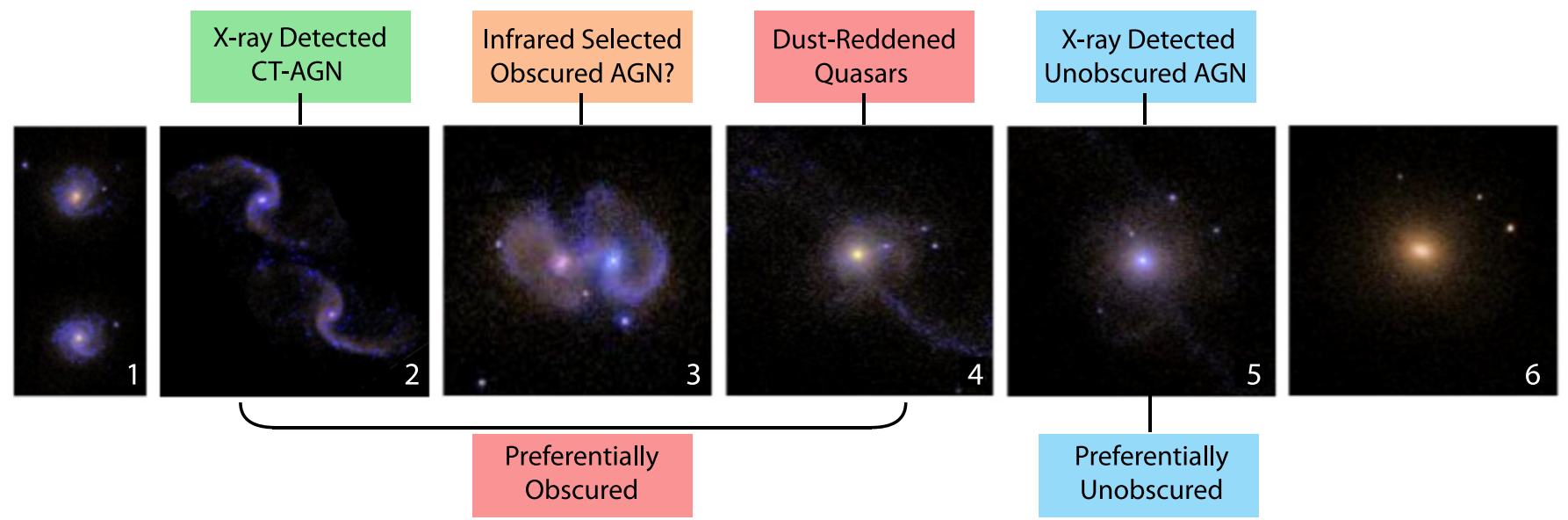

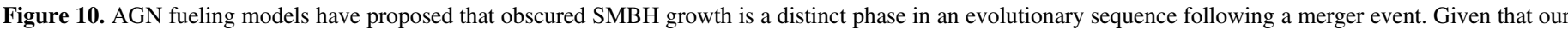

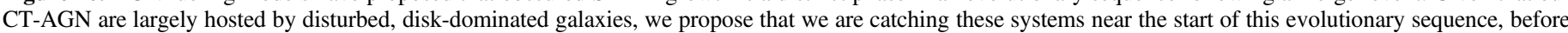

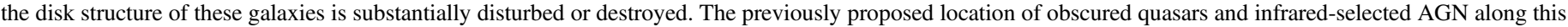
sequence is also shown. See Section 5.1 for details.

this scheme AGN obscuration is largely dependent on the viewing angle of the observer (Antonucci 1993; Urry \& Padovani 1995; Tran 2003) and therefore all AGN would sample the same parent population of host galaxies, regardless of their level of obscuration. In other words, there should be no correlation between heavy nuclear obscuration and disturbed host morphologies. Alternatively, obscured SMBH growth may be a distinct phase in the co-evolution of AGN and their hosts, specifically one in which the central engine goes through a rapid growth phase following a merger event (Sander et al. 1998; Hopkins et al. 2005, 2008). This is supported by the findings of Draper \& Ballantyne (2010), who suggest that a vast majority of AGN accreting near the Eddington limit must be hidden by Compton-thick obscuration based on the observed space density of CT-AGN and their contribution to the CXB. Furthermore, hydrodynamical merger simulations predict that this obscured phase should coincide with the most morphologically disturbed phase of a galaxy interaction (Cattaneo et al. 2005; Hopkins et al. 2008). Therefore, merger-driven coevolution models predict that there should be a strong dependence between obscuration and host properties such as morphology.

The fact that we observe a different disk/spheroid and merger fraction versus obscuration indicates that the viewing angle cannot be the only thing differentiating our three AGN samples, as the unification model would suggest. That is not to say that viewing angle plays no part in obscuring the CT-AGN in our sample, only that interactions play a greater role in fueling their activity relative to the unobscured AGN in our control samples. This finding appears to support an evolutionary scenario in which an increased fraction of the CT-AGN are heavily obscured as a result of a growth phase triggered by a galaxy interaction in the recent past. Given that the CT-AGN are hosted by largely disk-dominated galaxies, we propose that we are catching these systems near the start of this evolutionary sequence, before the disk structure of these galaxies is substantially disturbed or destroyed. This may be due to increasing obscuration levels as the merger sequence progresses. For example, if the covering fraction of the obscuring torus is higher for sources further along in the merger sequence, then the fraction of X-ray photons scattered into our line of sight would decrease and we would not be sensitive to the most disturbed sources. Our proposed location for the CT-AGN sample in a possible evolutionary sequence is illustrated in Figure 10.

Previous studies have reached similar conclusions regarding the transitional nature of obscured AGN. For example, the hosts of dust-reddened quasars (Glikman et al. 2004, 2012; Urrutia et al. 2009) show a high incidence of merger activity and a disturbance fraction that increases with increasing obscuration (Urrutia et al. 2008; Glikman et al. 2015). These quasars are intrinsically more luminous than the CT-AGN in our sample and are preferentially found in spheroid-dominated hosts. It is therefore thought that these quasars are detected in the final stages of emerging from their dusty cocoons and near the end of the evolutionary sequence outlined in Figure 10 (Banerji et al. 2012; Glikman et al. 2012; Urrutia et al. 2012).

Our results agree with the findings of local studies $(z<0.2)$ of obscured AGNs, namely Koss et al. (2010) and Satyapal et al. (2014), who report an elevated merger fraction for AGNs in the Swift BAT hard X-ray sample ${ }^{15}$ and an elevated pair frequency among WISE infrared-selected AGNs, respectively. At higher redshifts $(z \sim 1-2)$ our results also agree with the recent findings of J. L. Donley et al. (2015, in preparation) and Juneau et al. (2013), who examined the morphologies and starformation activity, respectively, of obscured AGN selected by their mid-infrared colors and emission line properties. In the former, the hosts of IRAC power-law selected AGN (Donley et al. 2008) are found to be more disturbed than their X-ray selected, and presumably less obscured, counterparts. In the latter, the obscured AGN fraction is found to be higher among galaxies with elevated specific star-formation rates, which the authors argue may be due to recent galaxy interactions.

On the other hand, our findings are at odds with the results of Schawinski et al. (2012). They examined the morphology of DOGs in the extended CDFS, which are thought to host heavily obscured quasars based on the X-ray stacking analysis of Treister et al. (2009). This study found that only a small fraction $(\sim 4 \%)$ of DOGs at $1<z<3$ show signs of recent merger activity. It is worth noting, however, that only one of our CT-AGN in the CDFS would be selected as an obscured

\footnotetext{
15 Although the Swift BAT sample does not preferentially select obscured AGNs, the 14-195 keV selection band allows for their unbiased detection.
} 
AGN via the infrared excess method employed by Schawinski et al. (2012). This is consistent with the findings of Comastri et al. (2011), who noted that X-ray detected CT-AGN are not readily picked up by standard mid-infrared selection techniques. We therefore suspect that our conflicting results are due to the substantial differences in our parent samples and caution against direct comparisons of the two studies.

\subsection{Implications for the AGN-merger Connection}

Galaxy mergers have long been proposed as a possible triggering mechanism for AGN activity; however, there is a growing consensus that most moderate-luminosity, X-ray selected AGN show no signs of recent merger activity based on their host morphologies (Grogin et al. 2005; Cisternas et al. 2011; Schawinski et al. 2011; Kocevski et al. 2012; Villforth et al. 2014). In fact, recent results from the CANDELS survey suggest that stochastic fueling by secular processes or disk instabilities play a greater role in fueling SMBH growth at $z>1$ than previously predicted by AGN fueling models (Kocevski et al. 2012). This is likely due to the increasing gas fraction of galaxies at high redshifts (e.g., Tacconi et al. 2010), which acts to increase the duty cycle of distant, stochastically fed AGN (Hopkins et al. 2007; Johansson et al. 2009).

However, even with these new observational constraints, AGN fueling models continue to predict that the integrated total SMBH growth in the universe should be dominated by merger-induced fueling. For example, the semi-emprical fueling model of Hopkins et al. (2014), which incorporates both stochastic and merger-induced fueling modes, finds that while non-merger processes may dominate the AGN population by numbers, only $\sim 30 \%$ of the total AGN luminosity density and SMBH mass density is the result of stochastic fueling. The predicted contribution is strongly mass and luminosity-dependent, with mergers playing an increasingly important role in fueling high-mass $\left(M_{\mathrm{BH}}>10^{7} M_{\odot}\right) \mathrm{SMBH}$ growth and high luminosity $\left(L_{\mathrm{bol}}>10^{12} L_{\odot}\right)$ AGN. A similar conclusion was reached by Draper \& Ballantyne (2012) using AGN population synthesis modeling to determine the importance of different AGN triggering mechanisms.

It is conceivable that some of the merger-induced fueling that is predicted may have been missed by past studies of the Chandra deep fields, given the few high luminosity AGN present in these fields (e.g., Triester et al. 2012). However, our results indicate that a portion of this merger-triggered activity may also be hidden among heavily obscured AGN. This implies that past studies may have missed the AGN population where the AGN-merger connection is expected to be the strongest. Whether there is sufficient merger-fueled SMBH growth occurring among heavily obscured and high luminosity AGN to match what is predicted by the Hopkins et al. (2014) fueling model remains to be determined. A key to testing this will be identifying additional CT-AGN at $z \sim 1-2$ in order to determine what fraction of this obscured growth remains undetected.

\subsection{Caveats and Future Work}

Having discussed the possible implications of our results, it is worth noting a couple important caveats. First, the difference in the disturbed fraction between the obscured and unobscured AGN is statistically significant only when point-like hosts are included in our analysis. When we consider only hosts with extended morphologies, the elevated merger fraction among the CT-AGN is significant at only the $2.5 \sigma$ level. A larger sample of distant, obscured AGN will need to be identified in order to confirm our findings with greater statistical confidence. This will soon be possible as a result of the X-UDS Chandra Legacy Survey (co-PIs G. Hasinger and D. Kocevski), which is obtaining deep $(1.25 \mathrm{~ms})$ and wide $\left(22^{\prime} \times 22^{\prime}\right)$ X-ray observations of the UKIRT Infrared Deep Sky Survey (UKIDSS) Ultra-deep Survey field (UDS; Cirasuolo et al. 2007; Lawrence et al. 2007). This data set, when combined with the existing ACS and WFC3 imaging from CANDELS, will substantially increase the number of CT-AGN at $z \sim 1$ available for study. It will also increase the number of CT-AGN at $z \sim 2$ that have rest frame optical imaging, allowing us to extend our study to this redshift for the first time.

The second caveat relates to a possible connection between the elevated disk and disturbed fractions among the CT-AGN. If morphological disturbances are easier to visually detect in late-type systems, then it is possible that the increased Disturbed/Asymmetric fraction among the CT-AGN is simply a reflection of their higher Disk fraction relative to the control samples. We do not believe this is the case, because the CTAGN also show an increased Merger/Interaction fraction, which is a classification that requires a visible interacting neighbor or a train-wreck morphology. In other words, highly disruptive events that should be detectable in early-type galaxies and their late-type counterparts. Nonetheless, further work is needed to determine what fraction of interactions may be missed among spheroidal hosts. The CANDELS team is actively pursuing this work by visually classifying simulated interactions using a classification scheme similar to the one used in this study.

\section{CONCLUSIONS}

We used HST/ACS imaging to examine the morphologies of galaxies hosting heavily obscured AGN at $z \sim 1$ in order to test whether obscured SMBH growth at this epoch is linked to major merger events. Using the X-ray spectral analysis of Brightman et al. (2014), we select 154 heavily obscured AGN with $N_{\mathrm{H}}>10^{23.5} \mathrm{~cm}^{-2}$ and $z<1.5$ in the CDFS, EGS, and COSMOS fields. To determine if these AGN are triggered by galaxy interactions more often than less obscured AGN, we construct two control samples composed of moderately obscured $\left(10^{22}<N_{\mathrm{H}}<10^{23.5}\right)$ and unobscured $\left(N_{\mathrm{H}}<10^{23.5}\right)$ AGN. These samples are matched in redshift and intrinsic X-ray luminosity to the heavily obscured AGN sample. To determine the morphology of the host galaxies, we employ a visual classification scheme similar to the one used in Kocevski et al. (2012) and by the CANDELS collaboration. We assess both the predominant morphology of each host galaxy and the level of disturbance that is visible. Based on our visual classifications, we find:

1. The heavily obscured AGN are predominantly hosted by late-type galaxies; $65.3_{-4.6}^{+4.1} \%$ are classified as Disks, while only $16.5_{-2.8}^{+3.9} \%$ are classified as Spheroids. This disk fraction is elevated relative to our control samples of moderately obscured and unobscured AGN, which have disk fractions of $50.9_{-3.0}^{+3.0} \%$ and $34.5_{-2.7}^{+2.9} \%$, respectively. All three samples have a low Irregular/Peculiar fraction, 
which ranges from $6.4_{-1.2}^{+1.8} \%$ for the unobscured AGN to $16.5_{-2.8}^{+3.9} \%$ for the most heavily obscured.

2. We find a statistically significant increase in the fraction of disturbed hosts versus AGN obscuration. Roughly $21.5_{-3.3}^{+4.2} \%$ of the Compton-thick AGN have highly disturbed host morphologies and fall in the Merger/ Interaction class. This is true for only $7.8_{-1.3}^{+1.9} \%$ of the unobscured AGN, a difference that is significant at the $3.8 \sigma$ level. This trend with obscuration remains when we include galaxies that exhibit any minor disturbance or asymmetry in their morphology. Here the Disturbed/ Asymmetric fraction increases from $21.0_{-2.2}^{+2.6} \%$ for the unobscured AGN to $34.1_{-2.7}^{+2.9} \%$ for the moderately obscured AGN and $43.0_{-4.4}^{+4.6 \%}$ for the Compton-thick sample. The statistical significance of this increase is $4.4 \sigma$.

3. We find that the incidence of Point-like morphologies is inversely proportional to obscuration, as might be expected if heavy nuclear obscuration is blocking emission from the central engine. To account for any biases this may introduce, we excluded all unresolved hosts from our samples and repeated our analysis. When considering only extended hosts, we find that the disk fraction of the three subsamples is in much better agreement, although the heavily obscured AGN are still found in disk hosts more often than their unobscured counterparts $\left(68.7_{-4.6}^{+4.0} \%\right.$ versus $\left.55.1_{-3.8}^{+3.6} \%\right)$. Furthermore, the correlation between merger fraction and obscuration is still evident when excluding point sources, although at a reduced statistical significance. The Merger/Interaction fraction increases from $12.5_{-2.1}^{+2.9} \%$ to $22.6_{-3.4}^{+4.3} \%$ for the unobscured and heavily obscured samples, respectively; a difference that is now significant at the $2.5 \sigma$ level. A similar trend is found for the Disturbed/Asymmetric fraction, which increases from $33.5_{-3.4}^{+3.7} \%$ among the unobscured AGN to $45.2_{-4.5}^{+4.7} \%$ for the Compton-thick sample. Here the statistical significance is $2.3 \sigma$.

The fact that we observe a different disk/spheroid fraction versus obscuration indicates that viewing angle cannot be the only thing differentiating our three AGN samples, as a simple unification model would suggest. The increased fraction of disturbed morphologies with obscuration would appear to support an evolutionary scenario in which Compton-thick AGN are a distinct phase where the central SMBH undergoes rapid, obscured growth following a merger/interaction event. Given that our heavily obscured AGN are hosted by diskdominated galaxies, we propose that we are catching these systems near the start of this evolutionary sequence, before their disk structure is destroyed. Our findings also suggest that some of the merger-triggered SMBH growth that is predicted by AGN fueling models may be hidden among heavily obscured, Compton-thick AGN, as previous studies of dustreddened quasars have proposed. That said, a larger sample of distant, obscured AGN will need to be studied in order to confirm our findings with greater statistical confidence, especially among extended AGN hosts. This will soon be possible as a result of the UDS Chandra Legacy Survey, which will allow us to extend this work to $z \sim 2$.

Support for Program number HST-GO-12060 was provided by NASA through a grant from the Space Telescope Science
Institute, which is operated by the Association of Universities for Research in Astronomy, Incorporated, under NASA contract NAS5-26555.

\section{REFERENCES}

Akylas, A., Georgakakis, A., Georgantopoulos, I., Brightman, M., \& Nandra, K. 2012, A\&A, 546, A98

Alexander, D. M., Bauer, F. E., Brandt, W. N., et al. 2003, AJ, 126, 539

Alexander, D. M., Bauer, F. E., Brandt, W. N., et al. 2011, ApJ, 738, 44

Antonucci, R. 1993, ARA\&A, 31, 473

Banerji, M., McMahon, R. G., Hewett, P. C., et al. 2012, MNRAS, 427, 2275

Barnes, J. E., \& Hernquist, L. E. 1991, ApJL, 370, L65

Barro, G., Pérez-González, P. G., Gallego, J., et al. 2011a, ApJS, 193, 13

Barro, G., Pérez-González, P. G., Gallego, J., et al. 2011b, ApJS, 193, 30

Bennert, N., Canalizo, G., Jungwiert, B., et al. 2008, ApJ, 677, 846

Brightman, M., \& Nandra, K. 2011, MNRAS, 413, 1206

Brightman, M., Nandra, K., Salvato, M., et al. 2014, MNRAS, 443, 1999

Brightman, M., \& Ueda, Y. 2012, MNRAS, 423, 702

Broos, P. S., Townsley, L. K., Feigelson, E. D., et al. 2010, ApJ, 714, 1582

Brusa, M., Civano, F., Comastri, A., et al. 2010, ApJ, 716, 348

Buchner, J., Georgakakis, A., Nandra, K., et al. 2014, A\&A, 564, A125

Buchner, J., Georgakakis, A., Nandra, K., et al. 2015, ApJ, 802, 89

Cameron, E. 2010, arXiv: 1012.0566

Capak, P., Aussel, H., Ajiki, M., et al. 2007, ApJS, 172, 99

Cardamone, C. N., van Dokkum, P. G., Urry, C. M., et al. 2010, ApJS, 189,270

Cash, W. 1979, ApJ, 228, 939

Cattaneo, A., Combes, F., Colombi, S., Bertin, E., \& Melchior, A.-L. 2005, MNRAS, 359, 1237

Cirasuolo, M., McLure, R. J., Dunlop, J. S., et al. 2007, MNRAS, 380, 585

Cisternas, M., Jahnke, K., Inskip, K. J., et al. 2011, ApJ, 726, 57

Civano, F., Brusa, M., Comastri, A., et al. 2011, ApJ, 741, 91

Civano, F., Elvis, M., Brusa, M., et al. 2012, ApJS, 201, 30

Comastri, A., Ranalli, P., Iwasawa, K., et al. 2011, A\&A, 526, L9

Comastri, A., Setti, G., Zamorani, G., \& Hasinger, G. 1995, A\&A, 296, 1

Cooper, M. C., Griffith, R. L., Newman, J. A., et al. 2012, MNRAS, 419, 3018

Daddi, E., Alexander, D. M., Dickinson, M., et al. 2007, ApJ, 670, 173

Davis, M., Guhathakurta, P., Konidaris, N. P., et al. 2007, ApJL, 660, L1

Di Matteo, T., Springel, V., \& Hernquist, L. 2005, Natur, 433, 604

Donley, et al. 2015, in preparation

Donley, J. L., Koekemoer, A. M., Brusa, M., et al. 2012, ApJ, 748, 142

Donley, J. L., Rieke, G. H., Pérez-González, P. G., \& Barro, G. 2008, ApJ, 687, 111

Donley, J. L., Rieke, G. H., Pérez-González, P. G., Rigby, J. R., \& Alonso-Herrero, A. 2007, ApJ, 660, 167

Draper, A. R., \& Ballantyne, D. R. 2010, ApJL, 715, L99

Draper, A. R., \& Ballantyne, D. R. 2012, ApJ, 751, 72

Ellison, S. L., Patton, D. R., Mendel, J. T., \& Scudder, J. M. 2011, MNRAS, 418,2043

Elvis, M., Civano, F., Vignali, C., et al. 2009, ApJS, 184, 158

Fabian, A. C. 1999, MNRAS, 308, L39

Feruglio, C., Daddi, E., Fiore, F., et al. 2011, ApJL, 729, L4

Fiore, F., Grazian, A., Santini, P., et al. 2008, ApJ, 672, 94

Gandhi, P., Lansbury, G. B., Alexander, D. M., et al. 2014, ApJ, 792, 117

Gebhardt, K., Bender, R., Bower, G., et al. 2000, ApJL, 539, L13

Georgantopoulos, I., Akylas, A., Georgakakis, A., \& Rowan-Robinson, M. 2009, A\&A, 507, 747

Georgantopoulos, I., Comastri, A., Vignali, C., et al. 2013, A\&A, 555, A43

Giavalisco, M., Ferguson, H. C., Koekemoer, A. M., et al. 2004, ApJL, 600, L93

Gilli, R., Comastri, A., \& Hasinger, G. 2007, A\&A, 463, 79

Gilli, R., Su, J., Norman, C., et al. 2011, ApJ, 730, 28

Glikman, E., Gregg, M. D., Lacy, M., et al. 2004, ApJ, 607, 60

Glikman, E., Simmons, B., Mailly, M., et al. 2015, ApJ, 806, 218

Glikman, E., Urrutia, T., Lacy, M., et al. 2012, ApJ, 757, 51

Grogin, N. A., Conselice, C. J., Chatzichristou, E., et al. 2005, ApJL, 627, L97

Grogin, N. A., Kocevski, D. D., Faber, S. M., et al. 2011, ApJS, 197, 35

Gültekin, K., Richstone, D. O., Gebhardt, K., et al. 2009, ApJ, 698, 198

Guo, Y., Ferguson, H. C., Giavalisco, M., et al. 2013, ApJS, 207, 24

Guyon, O., Sanders, D. B., \& Stockton, A. 2006, ApJS, 166, 89

Hernquist, L. 1989, Natur, 340, 687

Hopkins, P. F., \& Hernquist, L. 2009, ApJ, 698, 1550

Hopkins, P. F., Hernquist, L., Cox, T. J., et al. 2005, ApJ, 630, 705

Hopkins, P. F., Hernquist, L., Cox, T. J., \& Kereš, D. 2008, ApJS, 175, 356 
Hopkins, P. F., Hernquist, L., Cox, T. J., Robertson, B., \& Krause, E. 2007, ApJ, 669, 45

Hopkins, P. F., Hernquist, L., Cox, T. J., Robertson, B., \& Springel, V. 2006, ApJS, 163, 50

Hopkins, P. F., Kocevski, D. D., \& Bundy, K. 2014, MNRAS, 445, 823

Hsu, L.-T., Salvato, M., Nandra, K., et al. 2014, ApJ, 796, 60

Johansson, P. H., Burkert, A., \& Naab, T. 2009, ApJL, 707, L184

Juneau, S., Dickinson, M., Bournard, F., et al. 2013, ApJ, 764, 176

Kartaltepe, J. S., Mozena, M., Kocevski, D., et al. 2015, ApJS, 221, 11

Kauffmann, G., \& Haehnelt, M. 2000, MNRAS, 311, 576

Kocevski, D. D., Faber, S. M., Mozena, M., et al. 2012, ApJ, 744, 148

Koekemoer, A. M., Aussel, H., Calzetti, D., et al. 2007, ApJS, 172, 196

Koekemoer, A. M., Faber, S. M., Ferguson, H. C., et al. 2011, ApJS, 197, 36

Koss, M., Mushotzky, R., Treister, E., et al. 2012, ApJL, 746, L22

Koss, M., Mushotzky, R., Veilleux, S., \& Winter, L. 2010, ApJL, 716, L125

Lacy, M., Storrie-Lombardi, L. J., Sajina, A., et al. 2004, ApJS, 154, 166

Laird, E. S., Nandra, K., Georgakakis, A., et al. 2009, ApJS, 180, 102

Lansbury, G. B., Gandhi, P., Alexander, D. M., et al. 2015, ApJ, 809, 115

Lanzuisi, G., Civano, F., Elvis, M., et al. 2013, MNRAS, 431, 978

Lanzuisi, G., Ranalli, P., Georgantopoulos, I., et al. 2015, A\&A, 573, A137

Lawrence, A., Warren, S. J., Almaini, O., et al. 2007, MNRAS, 379, 1599

Lilly, S. J., Le Brun, V., Maier, C., et al. 2009, ApJS, 184, 218

Magorrian, J., Tremaine, S., Richstone, D., et al. 1998, AJ, 115, 2285

Matt, G., Brandt, W. N., \& Fabian, A. C. 1996, MNRAS, 280, 823

McConnell, N. J., \& Ma, C.-P. 2013, ApJ, 764, 184

Mihos, J. C., \& Hernquist, L. 1996, ApJ, 464, 641

Nandra, K., Laird, E. S., Aird, J. A., et al. 2015, ApJS, 220, 110

Newman, J. A., Cooper, M. C., Davis, M., et al. 2013, ApJS, 208, 5

Pierce, C. M., Lotz, J. M., Laird, E. S., et al. 2007, ApJL, 660, L19

Reynolds, C. S., Fabian, A. C., Makishima, K., Fukazawa, Y., \& Tamura, T. 1994, MNRAS, 268, L55

Robertson, B., Bullock, J. S., Cox, T. J., et al. 2006, ApJ, 645, 986
Rosario, D. J., McIntosh, D. H., van der Wel, A., et al. 2015, A\&A, 573, A85

Salvato, M., Ilbert, O., Hasinger, G., et al. 2011, ApJ, 742, 61

Sanders, D. B., Salvato, M., Aussel, H., et al. 2007, ApJS, 172, 86

Sanders, D. B., Soifer, B. T., Elias, J. H., et al. 1988, ApJ, 325, 74

Satyapal, S., Ellison, S. L., McAlphone, W., et al. 2014, MNRAS, 441,129

Schawinski, K., Simmons, B. D., Urry, C. M., Treister, E., \& Glikman, E. 2012, MNRAS, 425, L61

Schawinski, K., Treister, E., Urry, C., et al. 2011, ApJ, 727, 31

Silverman, J. D., Kampczyk, P., Jahnke, K., et al. 2011, ApJ, 743, 2

Soifer, B. T., Helou, G., \& Werner, M. 2008, ARA\&A, 46, 201

Springel, V., \& Hernquist, L. 2005, ApJ, 622, 9

Stern, D., Eisenhardt, P., Gorjian, V., et al. 2005, ApJ, 631, 163

Sutherland, W., \& Saunders, W. 1992, MNRAS, 259, 413

Tacconi, L. J., Genzel, R., Neri, R., et al. 2010, Natur, 463, 781

Tozzi, P., Gilli, R., Mainieri, V., et al. 2006, A\&A, 451, 457

Tran, H. D. 2003, ApJ, 583, 632

Treister, E., Cardamone, C. N., Schawinski, K., et al. 2009, ApJ, 706, 535

Treister, E., Schawinski, K., Urry, C. M., \& Simmons, B. D. 2012, ApJL, 758, L39

Treister, E., Urry, C. M., Chatzichristou, E., et al. 2004, ApJ, 616, 123

Tremaine, S., Gebhardt, K., Bender, R., et al. 2002, ApJ, 574, 740

Trump, J. R., Impey, C. D., Elvis, M., et al. 2009, ApJ, 696, 1195

Ueda, Y., Akiyama, M., Ohta, K., \& Miyaji, T. 2003, ApJ, 598, 886

Urrutia, T., Becker, R. H., White, R. L., et al. 2009, ApJ, 698, 1095

Urrutia, T., Lacy, M., \& Becker, R. H. 2008, ApJ, 674, 80

Urrutia, T., Lacy, M., Spoon, H., et al. 2012, ApJ, 757, 125

Urry, C. M., \& Padovani, P. 1995, PASP, 107, 803

Veilleux, S., Kim, D.-C., Rupke, D. S. N., et al. 2009, ApJ, 701, 587

Villforth, C., Hamann, F., Rosario, D. J., et al. 2014, MNRAS, 439, 3342

Xue, Y. Q., Luo, B., Brandt, W. N., et al. 2011, ApJS, 195, 10 